CUBO A Mathematical Journal

Vol.19, No 03, (69-77). October 2017

\title{
Totally Degenerate Extended Kleinian Groups
}

\author{
RuBÉN A. HidAlgo 1 \\ Departamento de Matemática y Estadística \\ Universidad de La Frontera, Temuco, Chile \\ ruben.hidalgo@ufrontera.cl
}

\begin{abstract}
The theoretical existence of totally degenerate Kleinian groups is originally due to Bers and Maskit. In fact, Maskit proved that for any co-compact non-triangle Fuchsian group acting on the hyperbolic plane $\mathbb{H}^{2}$ there is a totally degenerate Kleinian group algebraically isomorphic to it. In this paper, by making a subtle modification to Maskit's construction, we show that for any non-Euclidean crystallographic group $F$, such that $\mathbb{H}^{2} / F$ is not homeomorphic to a pant, there exists an extended Kleinian group $\mathrm{G}$ which is algebraically isomorphic to $\mathrm{F}$ and whose orientation-preserving half is a totally degenerate Kleinian group. Moreover, such an isomorphism is provided by conjugation by an orientation-preserving homeomorphism $\phi: \mathbb{H}^{2} \rightarrow \Omega$, where $\Omega$ is the region of discontinuity of G. In particular, this also provides another proof to Miyachi's existence of totally degenerate finitely generated Kleinian groups whose limit set contains arcs of Euclidean circles.
\end{abstract}

\section{RESUMEN}

La existencia teórica de grupos Kleinianos totalmente degenerados se debe originalmente a Bers y Maskit. De hecho, Maskit demostró que para cualquier grupo Fuchsiano co-compacto y no-triangular actuando en el plano hiperbólico $\mathbb{H}^{2}$ existe un grupo Kleiniano totalmente degenerado algebraicamente isomorfo a él. En este artículo, haciendo una modificación sutil a la construcción de Maskit, mostramos que para cualquier grupo cristalográfico no-Euclidiano $F$ tal que $\mathbb{H}^{2} / F$ no es homeomorfo a un pantalón, existe un grupo Kleiniano extendido $G$ que es algebraicamente isomorfo a $F$ y cuya mitad que preserva orientación es un grupo Kleiniano totalmente degenerado. Más aún, un tal isomorfismo está dado por la conjugación por un homeomorfismo que preserva orientación $\phi: \mathbb{H}^{2} \rightarrow \Omega$, donde $\Omega$ es la región de discontinuidad de G. En particular, esto

\footnotetext{
${ }^{1}$ Partially supported by Project Fondecyt 1150003 and Anillo ACT 1415 PIA-CONICYT
} 


\section{CUBO}

19, 3 (2017)

también entrega otra demostración del resultado de Miyachi acerca de la existencia de grupos Kleinianos totalmente degenerados finitamente generados cuyo conjunto límite contiene arcos de circunferencias Euclidianas.

Keywords and Phrases: Kleinian Groups, NEC groups

2010 AMS Mathematics Subject Classification: 30F40, 30F50 


\section{Introduction}

The classical uniformization theorem asserts that every non-exceptional Riemann surface $\mathrm{S}$, i.e., non-isomorphic to either the Riemann sphere $\widehat{\mathbb{C}}$, the complex plane $\mathbb{C}$, the puncture plane $\mathbb{C}-\{0\}$ or a torus, is conformally equivalent to a quotient $\mathbb{H}^{2} / \Gamma$, where $\Gamma \cong \pi_{1}(S, *)$ is a discrete group of conformal automorphisms of the hyperbolic plane $\mathbb{H}^{2}$. Similarly, every Klein surface (i.e., a real surface where the local change of coordinates are either conformal or anti-conformal) which are non-exceptionals (i.e., those whose orientation-preserving doble covers are non-exceptional Riemann surfaces) is di-analytically equivalent to a quotient $X=\mathbb{H}^{2} / F$, where $F \cong \pi_{1}^{\text {orb }}(X)$ is a discrete group of conformal and anti-conformal automorphisms of $\mathbb{H}^{2}$ (necessarilly containing anticonformal ones). If $\mathrm{X}$ is compact and $\mathrm{F}^{+}$is the index two subgroup of $\mathrm{F}$ consisting of its conformal elements, then $\mathrm{F}$ is called a non-euclidean crystallographic (NEC) group of algebraic genus equal to the genus of the closed Riemann surface $\mathrm{X}^{+}=\mathbb{H}^{2} / \mathrm{F}^{+}$. If $\mathrm{X}$ is homeomorphic to the closure of the complement of three disjoint closed discs on the Riemann sphere, then it is called a (compact) pant.

A finitely generated non-elementary Kleinian group with a non-empty connected and simplyconnected region of discontinuity is called totally degenerate. The theoretical existence of such groups, in the boundary of Teichmüller spaces of co-compact non-triangle Fuchsian groups, is by now well-know due to Bers [1] and Maskit [4, but it seems that there is no explicit example of such type of groups in the literature. There is a nice construction in [3, IX.G] due to Maskit, as an application of the Klein-Maskit combination theorems [5, 6, 7] to (theoretically) obtain a totally degenerate Kleinian group isomorphic to a given co-compact Fuchsian group $\mathrm{F}^{+}$, different from a triangle one (i.e., the uniformized orbifold is not the sphere with exactly there cone points). The idea was to observe the existence of some $\rho_{0} \in(0,+\infty)$ (which can be chosen in the complement of a suitable countable subset), an open arc $\mathrm{T}$ of the circle centered at the origin and radius $\rho_{0}$, with $\rho_{0} \in \mathrm{T}$, such that:

(i) for each $t \in T$, there is a quasifuchsian group $\mathrm{G}^{+}(\mathrm{t})$ and there is an isomorphism $\theta_{\mathrm{t}}: \mathrm{F}^{+} \rightarrow$ $\mathrm{G}^{+}(\mathrm{t})$

(ii) $\mathrm{G}^{+}\left(\rho_{0}\right)$ is a Fuchsian group, and

(iii) for one of the end points $\mathrm{t}^{\#}$ of $\mathrm{T}$, there is a totally degenerate Kleinian group $\mathrm{G}^{+}\left(\mathrm{t}^{\#}\right)$, with region of discontinuity $\Omega$, so that there is an orientation-preserving homeomorphism $\phi: \mathbb{H}^{2} \rightarrow \Omega$ inducing, by conjugation, an isomorphism between $\mathrm{F}^{+}$and $\mathrm{G}^{+}\left(\mathrm{t}^{\#}\right)$.

An extended Kleinian group is a group $\mathrm{G}$ of conformal and anticonformal automorphisms of the Riemann sphere, necessarily containing anticonformal elements, whose index two orientationpreserving half $\mathrm{G}^{+}$is a Kleinian group. In the case that $\mathrm{G}^{+}$is totally degenerate, we say that $\mathrm{G}$ is a totally degenerate extended Kleinian group. The existence of totally degenerate extended Kleinian groups should not be a surprise as, from a general abstract point of view, this follows 
from the non-triviality of the Teichmuller space of an orbifold with mirrored boundary. In this note we indicate the subtle modifications in Maskit's construction from [3, IX.G], to observe that for given a NEC group there is a totally degenerate Kleinian group isomorphic to it.

Theorem 1.1. Let $\mathrm{F}$ be a NEC group so that $\mathbb{H}^{2} / \mathrm{F}$ is not homeomorphic to a pant. Then there exists a totally degenerate extended Kleinian group $\mathrm{G}$, with region of discontinuity $\Omega$, and there exists an orientation-preserving homeomorphism $\phi: \mathbb{H}^{2} \rightarrow \Omega$ inducing, by conjugation, an isomorphic between $\mathrm{F}$ and $\mathrm{G}$.

If for the NEC group F it holds that $\mathbb{H}^{2} / \mathrm{F}$ has non-empty boundary, then Theorem 1.1 implies the following result due to Miyachi.

Corollary 1.2 (Miyachi [8]). There are totally degenerate Kleinian groups for which there exist arcs of Euclidean circles inside the limit set; these arcs connect fixed points of distinct parabolic transformations and/or connect the fixed points of the same hyperbolic transformation. These arcs of circles are dense in the limit set.

\section{Preliminaries}

\subsection{Möbius and extended Möbius transformations}

The conformal automorphisms of the Riemann sphere $\widehat{\mathbb{C}}$ are the Möbius transformations and its anti-conformal ones are the extended Möbius transformations (the composition of the standard reflection $J(z)=\bar{z}$ with a Möbius transformation). We denote by $\mathbb{M}$ the group of Möbius transformations and by $\widehat{\mathbb{M}}$ the group generated by $\mathbb{M}$ and J. Clearly, $\mathbb{M}$ is an index two subgroup of $\widehat{\mathbb{M}}$. If $\mathrm{K}$ is a subgroup of $\widehat{\mathbb{M}}$, then we set $\mathrm{K}^{+}:=\mathrm{K} \cap \mathbb{M}$.

Möbius transformations are classified into parabolic, loxodromic (including hyperbolic) and elliptic transformations. First, we need to observe that a non-trivial Möbius transformation has at least one fixed point and at most two of them. The parabolic ones are those having exactly one fixed point, elliptic ones are conjugated to rotations and loxodromic are conjugated to transformations of the form $z \mapsto r e^{i \theta} z$, where $r \in(0,1) \cup(1,+\infty)$ (if $e^{i \theta}=1$, then we call it hyperbolic). Similarly, extended Möbius transformations are classified into pseudo-parabolic (the square is parabolic), glide-reflection (the square is hyperbolic), pseudo-elliptic (the square is elliptic), reflection (of order two admitting a circle of fixed points on $\widehat{\mathbb{C}}$ ) and imaginary reflection (of order two and having no fixed points on $\widehat{\mathbb{C}})[\underline{3}$.

Each Möbius transformation $\gamma$ can be identified with a projective linear transformation

$$
\gamma=\left[\begin{array}{ll}
a & b \\
c & d
\end{array}\right] \in \operatorname{PSL}_{2}(\mathbb{C})
$$

and the square of its trace $\operatorname{tr}(\gamma)^{2}=(a+d)^{2}$ is well defined. If $\gamma$ is different from the identity transformation, then the following hold: 
(1) $\gamma$ is parabolic if and only if $\operatorname{tr}(\gamma)^{2}=4$.

(2) $\gamma$ is elliptic if and only if $\operatorname{tr}(\gamma)^{2} \in[0,4)$.

(3) $\gamma$ is loxodromic if and only if $\operatorname{tr}(\gamma)^{2} \notin[0,4]$ (hyperbolic ones correspond $\operatorname{to} \operatorname{tr}(\gamma)^{2} \in(4,+\infty)$ ).

\subsection{Kleinian and extended Kleinian groups}

A Kleinian group is a discrete subgroup of $\mathbb{M}$, and an extended Kleinian group is a discrete subgroup of $\widehat{\mathbb{M}}$ containing extended Möbius transformations.

If $\mathrm{G}$ is either a Kleinian or an extended Kleinian group, then its region of discontinuity $\Omega(\mathrm{G})$ is the open subset of $\widehat{\mathbb{C}}$ (which might be empty) formed by those points $p \in \widehat{\mathbb{C}}$ with finite G-stabilizer $G_{p}=\{\gamma \in G: \gamma(p)=p\}$ and for which there is an open set $U_{p}, p \in U_{p}$, such that $\gamma\left(U_{p}\right) \cap U_{p}=\emptyset$, for $\gamma \in G-G_{p}$. The complement $\Lambda(G)=\widehat{\mathbb{C}}-\Omega(G)$ is called the limit set of $G$. If the limit set is finite, then $\mathrm{G}$ is called elementary; otherwise, it is called non-elementary. If $\mathrm{G}$ is an extended Kleinian group, then both $\mathrm{G}$ and $\mathrm{G}^{+}$have the same region of discontinuity.

Basic examples of Kleinian groups are the following ones. A function group is a finitely generated Kleinian group $\mathrm{G}^{+}$with an invariant connected component $\Delta$ of its region of discontinuity.; in this case, $\Lambda\left(\mathrm{G}^{+}\right)=\partial \Delta$. A quasifuchsian group is a function group whose limit set is a Jordan loop. A $B$-group is a finitely generated function group with a simply connected invariant component of its region of discontinuity. If $\mathrm{G}^{+}$is a B-group, say with the simply-connected invariant connected component $\Delta$, then the Riemann mapping's theorem ensures the existence of a biholomorphism $\mathrm{F}: \mathbb{H}^{2} \rightarrow \Delta$. Then $\mathrm{F}^{-1} \mathrm{G}^{+} \mathrm{F}$ is a discrete group of automorphisms of $\mathbb{H}^{2}$ (a Fuchsian group). A parabolic transformation $\mathrm{L} \in \mathrm{G}^{+}$is called accidental if $\mathrm{F}^{-1} \circ \mathrm{L} \circ \mathrm{F}$ is hyperbolic.

Examples of extended Kleinian groups are the following ones. An extended function group is a finitely generated extended Kleinian group $G$ with an invariant connected component $\Delta$ of its region of discontinuity. In this case, $\Lambda(G)=\partial \Delta$ and $\mathrm{G}^{+}$is a function group (the converse of this last fact is not in general true). An extended quasifuchsian group is an extended function group whose limit set is a Jordan loop (so its orientation-preserving half is a quasifuchsian group). An extended B-group is a finitely generated extended Kleinian group with a simply connected invariant connected component of its region of discontinuity. We observe that the orientation-preserving half of an extended B-group is a B-group, but the converse is in general not true (see part (2) of the next result).

Lemma 2.1. If $\mathrm{G}$ is an extended Kleinian group whose orientation-preserving half $\mathrm{G}^{+}$is a $B$ group, then either (1) $\mathrm{G}$ is an extended B-group, or (2) $\mathrm{G}^{+}$is a quasifuchsian group and there is an element of $\mathrm{G}-\mathrm{G}^{+}$permuting both components of its region of discontinuity.

Proof. Since $\mathrm{G}^{+}$is finitely generated, so is G. Let $\Delta$ be a simply connected invariant component of $\mathrm{G}^{+}$. If $\mathrm{G}$ is not an extended B-group, then there is some $\gamma \in \mathrm{G}-\mathrm{G}^{+}$so that $\Delta^{\prime}=\gamma(\Delta)$ is another 
different simply connected component of $\mathrm{G}^{+}$. It follows that $\mathrm{G}^{+}$is necessarily a quasifuchsian group.

Remark 2.2. Let $\mathrm{G}$ be an extended B-group. Then the following properties are easy to see (just from the previous definitions).

(1) $\mathrm{G}$ is an extended quasifuchsian group if and only if $\mathrm{G}^{+}$is a quasifuchsian group.

(2) $\mathrm{G}$ is an extended totally degenerate group if and only if $\mathrm{G}^{+}$is totally degenerate;

(3) G has accidental parabolic transformations if and only if $\mathrm{G}^{+}$has accidental parabolic transformations.

The above remark permits us to see the following fact.

Lemma 2.3. Let $\mathrm{G}$ be a non-elementary extended B-group. Then either

(1) $\mathrm{G}$ is an extended quasifuchsian group, or

(2) $\mathrm{G}$ is an extended totally degenerate group, or

(3) G contains accidental parabolic transformations.

Proof. As $\mathrm{G}^{+}$is a non-elementary B-group, then either $\mathrm{G}^{+}$is a quasifuchsian group, or $\mathrm{G}^{+}$is a totally degenerate group, or $\mathrm{G}^{+}$contains accidental parabolic transformations. The result now follows from Remark 2.2 .

\subsection{The Klein-Maskit's combination theorem}

We next state a simple version of Klein-Maskit's combination theorems which is enough for us in this paper.

Theorem 2.4 (Klein-Maskit's combination theorem [5, 6, 7]).

(1) (Free products) Let $\mathrm{K}_{\mathbf{j}}$ be a (extended) Kleinian group with region of discontinuity $\Omega_{\mathfrak{j}}$, for $\mathfrak{j}=1,2$. Let $\mathcal{F}_{\mathbf{j}}$ be a fundamental domain for $\mathrm{K}_{\mathfrak{j}}$ and assume that there is a simple closed loop $\Sigma$, contained in the interior of $\mathcal{F}_{1} \cap \mathcal{F}_{2}$, bounding two discs $\mathrm{D}_{1}$ and $\mathrm{D}_{2}$, so that, for $\mathrm{j}=1,2$, the set $\Sigma \cup D_{j} \subset \Omega_{3-j}$ is precisely invariant under the identity in $\mathrm{K}_{3-\mathrm{j}}$. Then $\mathrm{K}=\left\langle\mathrm{K}_{1}, \mathrm{~K}_{2}\right\rangle$ is a (extended) Kleinian group, with fundamental domain $\mathcal{F}_{1} \cap \mathcal{F}_{2}$, which is the free product of $\mathrm{K}_{1}$ and $\mathrm{K}_{2}$. Every finite order element in $\mathrm{K}$ is conjugated in $\mathrm{K}$ to a finite order element of either $\mathrm{K}_{1}$ or $\mathrm{K}_{2}$. Moreover, if both $\mathrm{K}_{1}$ and $\mathrm{K}_{2}$ are geometrically finite, then $\mathrm{K}$ is so.

(2) (HNN-extensions) Let $\mathrm{K}_{0}$ be a (extended) Kleinian group with region of discontinuity $\Omega$, and let $\mathcal{F}$ be a fundamental domain for $\mathrm{K}_{0}$. Assume that there are two pairwise disjoint simple closed loops $\Sigma_{1}$ and $\Sigma_{2}$, both of them contained in the interior of $\mathcal{F}_{0}$, so that $\Sigma_{j}$ bounds a disc $\mathrm{D}_{j}$ such that 
$\left(\Sigma_{1} \cup D_{1}\right) \cap\left(\Sigma_{2} \cup D_{2}\right)=\emptyset$ and that $\Sigma_{j} \cup D_{j} \subset \Omega$ is precisely invariant under the identity in $\mathrm{K}_{0}$. If $\mathrm{T}$ is either a loxodromic transformation or a glide-reflection so that $\mathrm{T}\left(\Sigma_{1}\right)=\Sigma_{2}$ and $\mathrm{T}\left(\mathrm{D}_{1}\right) \cap \mathrm{D}_{2}=\emptyset$, then $\mathrm{K}=\left\langle\mathrm{K}_{0}, \mathrm{f}\right\rangle$ is a (extended) Kleinian group, with fundamental domain $\mathcal{F}_{1} \cap\left(\mathrm{D}_{1} \cup \mathrm{D}_{2}\right)^{\mathrm{c}}$, which is the HNN-extension of $\mathrm{K}_{0}$ by the cyclic group $\langle\mathrm{T}\rangle$. Every finite order element of $\mathrm{K}$ is conjugated in $\mathrm{K}$ to a finite order element of $\mathrm{K}_{0}$. Moreover, if $\mathrm{K}_{0}$ is geometrically finite, then $\mathrm{K}$ is so.

\section{Proof of Theorem 1.1}

We proceed to describe the main points of the arguments done in [4, IX.G], for the Fuchsian groups case, and the corresponding adaptation to the NEC groups case.

Let $F$ be a NEC group acting on the upper-half plane $\mathbb{H}^{2}$, such that $\mathbb{H}^{2} / \mathrm{F}$ is not a pant, and let $\pi: \mathbb{H}^{2} \rightarrow \mathbb{H}^{2} / \mathrm{F}$ be a di-analytic regular branched covering map induced by the action of $\mathrm{F}$.

As $\mathbb{H}^{2} / F$ is not a pant, we may choose a simple loop $w \subset \mathbb{H}^{2} / F$ so that each of its lifted arcs in $\mathbb{H}^{2}$, under $\pi$, has as F-stabilizer a cyclic group generated by a hyperbolic element being primitive (that is, it is not a non-trivial power of an element of $F$ ). Let $A \subset \mathbb{H}^{2}$ be one of the arcs in $\Sigma:=\pi^{-1}(\mathcal{w})$ and let $J=\langle j\rangle$ be its $F$-stabilizer (so $j$ is a primitive hyperbolic transformation). Note that the connected components of $\mathbb{H}^{2}-\Sigma$ are planar regions. Let $E_{1}$ and $E_{2}$ be the two of these regions containing $A$ on their borders and, for $m \in\{1,2\}$, let $F_{m}$ be the F-stablizer of $E_{m}$. As $E_{m}$ is precisely invariant under $F_{m}$ in $F$, it follows that $E_{m} / F_{m}$ is embedded in $\mathbb{H}^{2} / F$. Moreover, $\mathbb{H}^{2} / F$ is the union of $E_{1} / F_{1}, E_{2} / F_{2}$ and $w$. As $E_{m} / F_{m}$ is topologically finite, $F_{m}$ is finitely generated. As a consequence of the first Klein-Maskit combination theorem [5] it holds that

$$
\mathrm{F}=\left\langle\mathrm{F}_{1}, \mathrm{~F}_{2}\right\rangle=\mathrm{F}_{1} *_{\mathrm{J}} \mathrm{F}_{2} .
$$

We may normalize $F$ so that $A$ is contained in the imaginary line, that is, $j$ has its fixed points at 0 and $\infty$. We assume that $E_{1}$ contains positive real points on its border.

If $t \in \mathbb{C}-\{0\}$ and $k_{t}(z)=t z$, then let $G(t)=\left\langle F_{1}, F_{2}(t)\right\rangle$, where $F_{2}(t)=k_{t} F_{2} k_{t}^{-1}$. For each $t$ there is a natural surjective homomorphism $\phi_{\mathrm{t}}: \mathrm{F} \rightarrow \mathrm{G}(\mathrm{t})$, defined as the identity on $\mathrm{F}_{1}$, and as the isomorphism $f \mapsto k_{t} \circ f \circ k_{t}^{-1}$ on $F_{2}$. Clearly, this restricts to a surjective homomorphism $\phi_{t}: F^{+} \rightarrow$ $\mathrm{G}(\mathrm{t})^{+}$. We should note that, for $\mathrm{t} \in(1,+\infty)$, the group $\mathrm{G}(\mathrm{t})$ is a group of conformal and anticonformal automorphisms of $\mathbb{H}^{2}$, in particular, asserting that $\mathrm{G}(\mathrm{t})$ is a NEC group topologically conjugated to F.

In [4, Lemma G.6]) it was noted that, for every $f \in \mathrm{F}^{+}$, the fixed points, and the square of the trace of $\phi_{t}(f)$, are holomorphic functions of $t \in \mathbb{C}-\{0\}$. As a consequence, for each hyperbolic $f \in \mathrm{F}^{+}$, there are only countable many complex numbers $t$ so that $\phi_{t}(f) \in G(t)^{+}$is parabolic. Since $\mathrm{F}^{+}$is countable, there are only countably many $\rho>0$ for which there is a $t=\rho e^{i \theta}$ so that for some hyperbolic $f \in F^{+}, \phi_{t}(f)$ is parabolic. So, we may find $\rho=\rho_{0}$ so that $\operatorname{tr}^{2}\left(\phi_{t}(f)\right) \neq 4$ for all hyperbolic $f \in F^{+}$and for all $t=\rho_{0} e^{i \theta}$. 
We know from the above that $G\left(\rho_{0}\right)$ is still a NEC group and, for $\theta$ small, the group $G\left(\rho_{0} e^{i \theta}\right)$ is an extended quasifuchsian group.

Similarly as done for the Fuchsian situation, we let $\mathrm{T}$ be the set of complex number of the form $t=\rho_{0} e^{i \theta}$ for which there is a loop $W(t)$ dividing $\widehat{\mathbb{C}}$ into two closed discs, $B_{1}(t)$ and $B_{2}(t)$, where $B_{1}(t)$ is a $\left(J, F_{1}\right)$-block (see [4, VII.B.4]) and $B_{2}(t)$ is precisely invariant under $J$ in $F_{2}(t)$. Note that: (i) $\rho_{0} \in T$, in this case $B_{1}=B_{1}\left(\rho_{0}\right)$ is the left half-plane and $B_{2}=B_{2}\left(\rho_{0}\right)$ is the right half-plane, and (ii) for small values of $\theta, \rho_{0} e^{i \theta} \in T$.

If $t \in T$, then the groups $F_{1}$ and $F_{2}(t)$ satisfy the hypothesis to use the first Klein-Maskit combination theorem, so $G(t)=F_{1} *_{J} F_{2}(t)$ and $G(t)$ is an extended quasifuchsian group so that $\phi_{t}: F \rightarrow G(t)$ is in an isomorphism. Also, there is a homeomorphism $\psi_{t}: \Omega(F) \rightarrow \Omega(G(t))$ inducing the isomorphism $\phi_{t}: F \rightarrow G(t)$ and so that $W(t)$ is the image under $\psi_{t}$ the circle given as the union of the imaginary line with $\infty$. Note that $B_{1}(t)$ is the disc containing $\psi_{t}\left(E_{2}\right)$ and $B_{2}(t)$ is the disc containing $\psi_{t}\left(E_{1}\right)$. The limit set $\Lambda(G(t))$ is a simple close curve passing through 0 and $\infty$; the complement, $\Omega(\mathrm{G}(\mathrm{t}))$ has two components, the upper component $\Delta(\mathrm{t})$ and the lower component $\Delta^{\prime}(\mathrm{t})$.

As a consequence of [3, Proposition IX.G.8], applied to the Fuchsian group $\mathrm{F}^{+}$and the quasifuchsian group $\mathrm{G}(\mathrm{t})^{+}$, one obtains that, if $\mathrm{t} \in \mathrm{T}$ and $\operatorname{Im}(\mathrm{t})>0$, then $\mathbb{H}^{2} \subset \Delta(\mathrm{t})$. Also, as observed in [4, IX.G.9], by interchanging the roles of $F_{1}$ and $F_{2}(t)$ it permits to observe that $\Delta(t)$ contains the half-plane $\{\arg (\mathrm{t})<\arg (z)<\arg (\mathrm{t})+\pi\}$. Applying [3, Lemma IX.G.10] to $\mathrm{F}^{+}$and $\mathrm{G}(\mathrm{t})^{+}$, it can be seen that $T$ is an open arc of the circle of radius $\rho_{0}$ with centre at the origin.

Next, we follow [3, IX.G.12]. We start at the point $t_{0}=\rho_{0} \in T$ and traverse counterclockwise to reach some first point $t^{\#}$ not in $T$; and we set $T_{0}$ be the $\operatorname{arc}$ of $T$ between $t_{0}$ and $t^{\#}$ (as noted in there, as $t$ traverses $T_{0}$ counterclockwise, from $t_{0}$ to $t^{\#}$, the upper component $\Delta(t)$ gets larger and the lower component $\Delta^{\prime}(t)$ gets smaller). Next, we fix a fundamental polygon $P_{1} \subset \mathbb{H}^{2}$ for $F_{1}$ and a fundamental polygon $P_{2} \subset \mathbb{H}^{2}$ for $F_{2}$. We choose $P_{1}$ and $P_{2}$ so that they are both contained in some fundamental polygon $E$ for $J$. Leave $P_{1}$ fixed and define $P_{2}(t)=k_{t}\left(P_{2}\right)$. So $P_{2}(t)$ is a fundamental polygon for $F_{2}(t)$ in the appropriate half-plane. The union $P_{1} \cup P_{2}(t)$ bounds a fundamental domain $\mathrm{D}(\mathrm{t}) \subset \Delta(\mathrm{t})$ for $\mathrm{G}(\mathrm{t})$ acting on $\Delta(\mathrm{t})$ and there is a homeomorphism $\psi_{\mathrm{t}}: \mathbb{H}^{2} \rightarrow \Delta(\mathrm{t})$ so that $\psi_{\mathrm{t}}\left(\mathrm{D}\left(\mathrm{t}_{\mathrm{o}}\right)\right)=\mathrm{D}(\mathrm{t})$ inducing the isomorphism $\phi_{\mathrm{t}}: \mathrm{F} \rightarrow \mathrm{G}(\mathrm{t})$.

It follows from the construction that, as $t \in T_{0}$ approaches $t^{\#}, \phi_{t}: F \rightarrow G(t)$ converges to a homomorphism $\phi: F \rightarrow G\left(t^{\#}\right)$. By our choice on $\rho_{0}$, it follows from 2] that $\phi: \mathrm{F}^{+} \rightarrow \mathrm{G}\left(\mathrm{t}^{\#}\right)^{+}$is type-preserving isomorphism.

Lemma 3.1. $\phi: \mathrm{F} \rightarrow \mathrm{G}\left(\mathrm{t}^{\#}\right)$ is an isomorphism.

Proof. Otherwise, there should be some $f \in F-F^{+}$so that $\phi(f)=1$. But, as $f^{2} \in F^{+}$and that $\phi: \mathrm{F}^{+} \rightarrow \mathrm{G}\left(\mathrm{t}^{\#}\right)^{+}$is an isomorphism, then $\mathrm{f}^{2}=1$. As $f$ is an anticonformal involution, $\phi_{\mathrm{t}}(\mathrm{f}) \in \mathrm{G}(\mathrm{t})$ is an anticonformal involution; so $\phi_{\mathrm{t}}(\mathrm{f})$ cannot approach the identity as $\mathrm{t}$ approaches $\mathrm{t}^{\#}$; a contradiction. 
Also, as $t \in T_{0}$ approaches $t^{\#}, \psi_{t}: D\left(t_{0}\right) \rightarrow \psi_{t}\left(D\left(t_{0}\right)\right) \subset \Delta(t)$ converges to a homeomorphism from $\mathrm{D}\left(\mathrm{t}_{\mathrm{o}}\right)$ onto its image D. Now, the same proof as [3, Lemma IX.G.13] permits to obtain that $G=G\left(t^{\#}\right)$ is an extended Kleinian group and that $D$ is precisely invariant under the identity in G. Working with $\mathrm{F}^{+}, \mathrm{G}(\mathrm{t})^{+}$and $\mathrm{G}^{+}$, we obtain (from [3, IX.G.14]) that $\mathrm{G}^{+}$is a B-group with a simply connected invariant component $\Delta$, where $\Delta / \mathrm{G}^{+}$is a finite Riemann surface homeomorphic to $\mathbb{H}^{2} / \mathrm{F}^{+}$. As consequence of [3, Proposition IX.G.15] it follows that $\mathrm{G}^{+}$is not quasifuchsian. Combining [3, IX. G.14], Lemma 2.1 and the above, one obtains that $\mathrm{G}$ is an extended B-group, different from a quasifuchsian one. As $\rho_{0}$ was constructed so that, for every $f \in F^{+}$hyperbolic and every $t=\rho_{0} e^{i \theta}$, the element $\phi_{t}(f) \in G^{+}(t)$ is not parabolic, it follows that $\phi(f)$ is neither parabolic. Hence the only elements of $\mathrm{G}^{+}(\mathrm{t})$ that are parabolic are conjugates of the parabolic elements of $\mathrm{F}_{1}^{+}$or $\mathrm{F}_{2}(\mathrm{t})^{+}$. But, as seen in [4, IX.G.14], they represent punctures on $\Delta / \mathrm{G}^{+}(\mathrm{t})$, so they are not accidental. Now, as consequence of all the above, together Lemma 2.3, we obtain that the group $\mathbf{G}\left(\mathfrak{t}^{\#}\right)$ is an extended totally degenerate group as desired.

\section{References}

[1] L. Bers. On boundaries of Teichmüller spaces and on Kleinian groups: I. Ann. of Math. 91 (1970), 570-600.

[2] V. Chuckrow. On Schottky groups with applications to Kleinian groups. Ann. of Math. 88 (1968), 47-61.

[3] B. Maskit, Kleinian Groups, GMW, Springer-Verlag, 1987.

[4] B. Maskit. On boundaries of Teichmüller spaces and on Kleinian groups: II. Ann. of Math. 91 (1970), 607-639.

[5] B. Maskit. On Klein's Combination Theorem Trans. of the Amer. Math. Soc. 120, No. 3 (1965), 499-509.

[6] B. Maskit. On Klein's combination theorem III. Advances in the Theory of Riemann Surfaces (Proc. Conf., Stony Brook, N.Y., 1969), Ann. of Math. Studies 66 (1971), Princeton Univ. Press, 297-316.

[7] Maskit, B. On Klein's combination theorem. IV. Trans. Amer. Math. Soc. 336 (1993), 265-294.

[8] H. Miyachi. Quasi-arcs in the limit set of a singly degenerate group with bounded geometry. In Kleinian Groups and Hyperbolic 3-Manifolds (Eds. Y.Komori, V.Markovic C.Series) LMS. Lec. Notes 299 (2003), 131-144. 